zwei Info-Veranstaltungen mit je 300 Besuchern abgehalten und auch eine Broschüre für Patienten und Ärzte erarbeitet. Beim Verlag C. H. Beck wurde die Lizenz für Formulare eingekauft, die die informierten Bürger dann nur noch ausfüllen und von ihrem Hausarzt abstempeln lassen müssen - eine große Erleichterung bei diesem leidigen Thema.

\section{Stark in der Weiterbildung}

Gemeinsam mit dem Klinikum Frankfurt-Höchst hat das GNEF einen Weiterbildungsverbund für die Facharztausbildung zum Arzt für Allgemeinmedizin gegründet. Koch und Kollegen erhoffen sich so auch Vorteile bei der Nachbesetzung von Arztsitzen. „Das ist nicht nur ein ländliches Problem“, erklärt sie. „Auch in den Randbezirken der Großstädte steht uns in den nächsten Jahren ein enormer Wechsel vor allem an Hausärzten bevor.“ Die Netz-Ärzte haben natürlich die Hoff- nung, dass die jungen Kollegen langfristig in der Region bleiben.

Netz-Managerin Müller macht sich vor allem für die Fort- und Weiterbildung der Fachangestellten stark. „Sie sind die ersten Ansprechpartnerinnen für die Patienten und müssen den Qualitätsgedanken ebenso verinnerlichen wie die Ärzte“, erklärt sie. Außer den standardisierten Schulungen in Datenschutz, Hygiene und Notfall biete man ihnen z. B. Fortbildungen zum Diabetischen Fuß an. An jeder Schulung nähmen etwa 30 MFA teil. Jede Praxis kann beliebig viele MFA schicken.

Für die Ärzte wiederum gibt es neben den DMP-Fortbildungen Veranstaltungen zu Themen wie Arbeitsrecht und Datenschutz oder den Neuerungen des Versorgungsstärkungsgesetzes. „All das, was den Ärzten in der Praxis auf den Nägeln brennt", sagt Koch.

\section{Aktuelle Urteile}

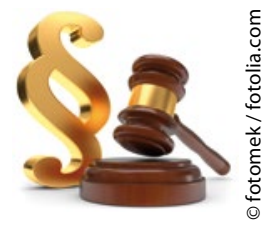

Årzte und Rechtsanwälte düirfen sich zusammentun

Das Bundesverfassungsgericht hat Partnerschaftsgesellschaften von Ärzten und Apothekern mit Rechtsanwälten erlaubt. Das war bisher in der Bundesrechtsanwaltsordnung verboten, was laut den Richtern mit dem Grundrecht der Berufsfreiheit unvereinbar ist. Der Gesetzgeber dürfe berufliche Zusammenschlüsse von Rechtsanwälten zwar begrenzen, doch tangiere eine Partnerschaft, die für Gutachten und Beratungen im Bereich des Arztrechts geschlossen wird, nicht die "anwaltlichen Grundpflichten" der Verschwiegenheit und Unabhängigkeit. Solchen Beratungsgesellschaften steht nun also nichts mehr im Wege.

- mwo/chy

Pete Smith

\title{
Mit der Pflege Hand in Hand
}

\author{
Am 8. und 9. April öffnet der Interprofessionelle Gesundheitskongress von Springer \\ Pflege in Dresden seine Porten. Mehr als 800 Ärzte, Pflegefachkräfte und Wissen- \\ schaftler diskutieren hier die Zukunft der gemeinsamen Patientenversorgung.
}

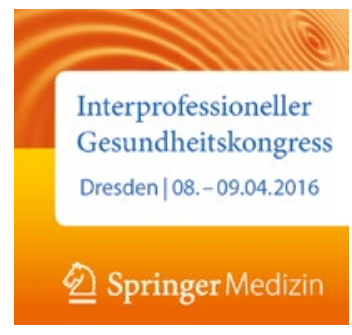

_ Eine gute Zusammenarbeit zwischen den Gesundheitsberufen sorgt für bessere Patientenergebnisse und erhöht die Berufszufriedenheit, wie Studien zeigen. Deshalb ist eine optimale Kooperation zwischen Ärzten, Pflegefachkräften und Medizinischen Fachangestellten (MFA) ein Ziel des Interprofessionellen Gesundheitskongresses von Springer Pflege, der am 8. und 9. April 2016 in Dresden stattfindet.

„Ein wichtiges Anliegen von mir ist es, den interdisziplinären Austausch zwischen Ärzten und Gesundheitsfachberufen zu fördern", sagt Erik Bodendieck, Präsident der Sächsischen Landesärztekammer (SLÄK). „Denn darin liegt für mich die Zukunft einer qualitativ hochwertigen Versorgung der Patienten." Die
SLÄK unterstützt seit vielen Jahren den Kongress, zu dem mehr als 800 Teilnehmer erwartet werden - darunter Ärzte, Pflegefachkräfte aller Versorgungsbereiche, Pflegemanager, MFAs und Gesundheitswissenschaftler.

\section{"Alle betreuen denselben Patienten“} Jana Luntz, Pflegedirektorin am Universitätsklinikum Dresden, weist darauf hin, dass ein interprofessioneller Ansatz der Logik der Patientenbehandlung folgt: „Schließlich arbeiten alle Berufsgruppen mit denselben Patienten“, so die Pflegemanagerin. Onkologie, Diabetes, der ältere Patient im Akutkrankenhaus, Arzneimitteltherapiesicherheit im Altersheim, Flüchtlingsversorgung im interprofessionellen Team - unter dem
Motto „Viele Professionen - ein Patient“ wartet der Kongress mit aktuellen und praxisnahen Schnittstellenthemen auf. Neben den interprofessionellen Veranstaltungen gibt es auch spezielle Module für Ärzte, etwa in der Kompressionstherapie oder der Reanimation. MFA können eigene Kurse etwa zu Impfen und Suchtprävention besuchen.

Wegen steigender Teilnehmerzahlen ist der Kongress in das Internationale Congress Center Dresden umgezogen, das direkt am Elbufer liegt. Neben der SLÄK und dem Universitätsklinikum Dresden sind u. a. der Bundesverband Pflegemanagement und der Sächsische Pflegerat Kooperationspartner.

\footnotetext{
$\rightarrow$ www.gesundheitskongresse.de/
} dresden/2016 